\title{
Artificially controlled degradable nanoparticles for contrast switch MRI and programmed cancer therapy
}

This article was published in the following Dove Press journal: International Journal of Nanomedicine

\author{
Tianyang Yun' \\ Yuxin $\mathrm{Liu}^{2}$ \\ Shaoqiong $\mathrm{Yi}^{1}$ \\ Qi Jia ${ }^{2}$ \\ Yang Liu' \\ Jing Zhou ${ }^{2}$
} Chinese People's Liberation Army General Hospital, Beijing I00853, People's Republic of China; ${ }^{2}$ Department of Chemistry, Capital Normal University, Beijing 100048,

People's Republic of China
'Department of Thoracic Surgery,
Correspondence: Yang Liu Department of Thoracic Surgery, Chinese People's Liberation Army General Hospital, Beijing 100853 ,

People's Republic of China

$\mathrm{Tel} / \mathrm{fax}+861066938313$

Email sunny30 Ix@sina.com

Jing Zhou

Department of Chemistry, Capital

Normal University, Beijing 100048,

People's Republic of China

Tel/fax +86 I0 6890 249I

Email jingzhou@cnu.edu.cn
Background: Utilizing the permeability enhancement and irreversible biomolecule denaturation caused by hyperthermia, photothermal-chemo synergistic therapy has shown great potential in clinical cancer treatment.

Purpose: The objective of this study was to provide a novel controlled drug release method to improve the efficiency of photothermal-chemo synergistic therapy.

Patients and methods: HCT116 tumor-bearing mice were selected as modal for the study of cancer theranostics efficiency. The $\mathrm{T} 2$ to $\mathrm{T} 1$ magnetic resonance imaging contrast switch was studied in vivo. Analyses of the tumor growth of mice were carried out to evaluate the tumor therapy efficiency.

Results: We developed novel artificially controlled degradable $\mathrm{Co}_{3} \mathrm{O}_{4}$ nanoparticles and explored their potential in drug delivery/release. In the presence of ascorbic acid (AA), the designed nanomaterials can be degraded via a redox process and hence release the loaded drugs. Importantly, the AA, in the lack of l-gulonolactone oxidase, cannot be synthesized in the body of typical mammal including human, which suggested that the degradation process can be controlled artificially. Moreover, the obtained nanoparticles have outstanding photothermal conversion efficiency and their degradation can also result in an magnetic resonance imaging contrast enhancement switch from $\mathrm{T}_{2}$ to $\mathrm{T}_{1}$, which benefits the cancer theranostics.

Conclusion: Our results illustrated that the artificially controlled degradable nanoparticles can serve as an alternative candidate for controllable drug release as well as a platform for highly efficient photothermal-chemo synergistic cancer theranostics.

Keywords: photothermal, degradable, controllable drug release, synergistic therapy, MRI

\section{Introduction}

With high surface area and outstanding biocompatibility, inorganic nanomaterials have propelled the development of the next-generation cancer chemotherapy as a carrier for drug delivery. ${ }^{1-4}$ As a result of the thorough exploration, highly efficient drug delivery can be practically achieved by constructing suitable nanocarriers. ${ }^{5-8}$ However, owing to the strong cytotoxicity of typical chemo drugs with low selectivity, developing a method for much more specific controlled drug release becomes the priority to the exploration of drug delivery system.

Recent advances emphasize the importance of achieving controlled drug release with the aid of specific tumor microenvironment, including low $\mathrm{pH}$ and imbalanced redox homeostasis. ${ }^{9-13}$ Therefore, the $\mathrm{pH}$ or redox degradable nanomaterials show promise as nanocarriers for drug delivery. As a proof of this concept, many phosphate-based and sulfide-based degradable nanocarriers have been developed and practiced in small animal models. ${ }^{14,15}$ Because photothermal therapy has efficient synergistic effect on chemotherapy, recent studies have further explored the 
degradable oxide-based semiconductor photothermal conversion nanomaterials for controlled drug release. ${ }^{16-19}$ The next step in the development of degradable carriers should focus on artificial control, instead of mainly depending on the specific microenvironment. Our recent work illustrates a novel manganese oxide-based nanomaterial for imagingguided photothermal therapy. ${ }^{20}$ It is noteworthy that this designed nanomaterial is stable in tumor microenvironment and normal biological fluids, but can be degraded in the presence of ascorbic acid (AA). The AA, as a result of L-gulonolactone oxidase deficiency, has to be introduced into the living system artificially, which provides an excellent strategy for controllable rapid post-therapy metabolism and detoxicification. ${ }^{21,22}$ More importantly, the decomposition of nanomaterial structure, which efficiently breaks the bondage between nanomaterials and loaded drugs, can also provide a new sight for controllable drug release. However, the controllable drug release using these artificially controlled degradable nanomaterials has not been studied, and even lesser their behavior and therapeutic efficiency in vivo. Therefore, it is necessary to develop and study the feasibility of artificially controlled degradable nanocarriers for drug delivery and controllable release, as well as for a synergistic cancer therapy in vivo.

Apart from the therapy, the nanocarriers should be equipped with imaging capacities to monitor the behavior and drug delivery/release in vivo. Among all the imaging methods, magnetic resonance imaging (MRI) can provide high spatial resolution images containing detailed soft tissue and pathological phenomena information. ${ }^{23-26}$ In addition, magnetic transition metal oxide nanomaterials, such as cobalt oxide and nickel oxide, are favorable $\mathrm{T}_{2}$-weighted MRI contrast agent by affecting transversal relaxation time. ${ }^{27,28}$ Moreover, the released transition metal ions within the process of degradation are very efficient $T_{1}$ contrast agents. ${ }^{29,30}$ According to previous reports, $\mathrm{Co}_{3} \mathrm{O}_{4}$ nanoparticles were feasible for MRI as $\mathrm{T}_{2}$ contrast agent, while the ionic degradation product $\mathrm{Co}^{2+}$ had potential for $\mathrm{T}_{1}$-weighted MRI. ${ }^{31,32}$ Thus, the designed degradable $\mathrm{Co}_{3} \mathrm{O}_{4}$-based nanocarriers can potentially offer a very useful technique to monitor the controlled degradation and drug release simultaneously by the time-dependent $\mathrm{T}_{2}$ to $\mathrm{T}_{1}$ contrast switching.

In this work, artificially controlled degradable cobalt oxide nanoparticles are successfully developed. By studying their AA-induced controllable degradation, drug delivery/ release, photothermal conversion, and magnetic resonance properties, the feasibilities of nanomaterial for further application are demonstrated. More significantly, the above properties, as well as time-dependent MRI contrast switch and systematic toxicity, are confirmed on the tumor-bearing mice model to illustrate their capacity for actual bioapplication. Our results provide a new sight for developing applicable inorganic nanocarriers toward drug delivery and synergistic cancer therapy (Scheme 1).

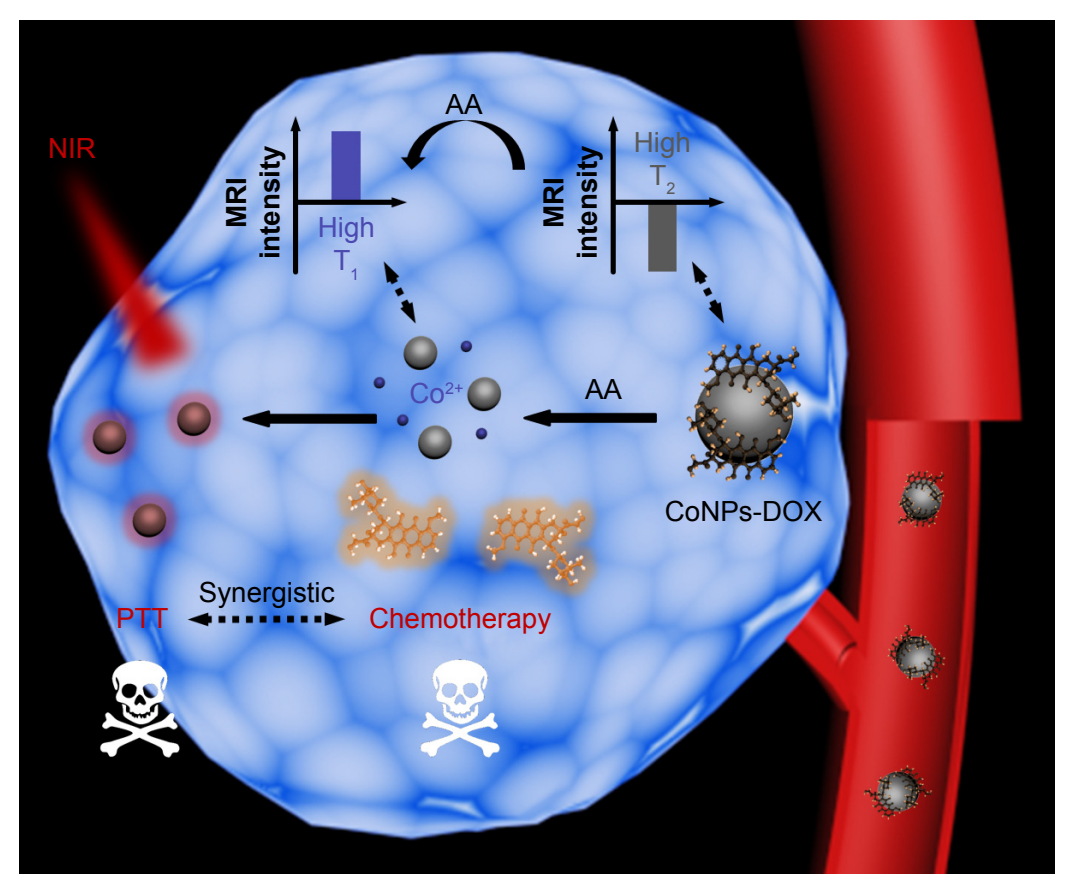

Scheme I Proposed application of artificially controlled degradable cobalt oxide nanoparticles for $T_{2}$ to $T_{1}$ contrast switch MRI-guided photothermal-chemo synergistic cancer therapy.

Abbreviations: CoNPs-DOX, $\mathrm{Co}_{3} \mathrm{O}_{4}$ nanoparticles-doxorubicin; NIR, near infrared; PTT, photothermal therapy; AA, ascorbic acid. 


\section{Materials and methods Materials}

$\mathrm{Co}(\mathrm{acac})_{2}$, AA, polyallylamine hydrochloride (PAH), polyacrylic acid (PAA), and oleylamine (OM) were purchased from Energy Chemical Co. Ltd, Shanghai, People's Republic of China. Ethanol, cyclohexane, citric acid (CA) hydrate, and dichloromethane $\left(\mathrm{CH}_{2} \mathrm{Cl}_{2}\right)$ were purchased from Beijing Chemical Reagent Company, Beijing, People's Republic of China. Oleic acid (OA) and nitronium tetrafluoroborate $\left(\mathrm{NOBF}_{4}\right)$ were purchased from Sigma Aldrich, Shanghai, People's Republic of China. 1-octadecene (ODE) was purchased from Alfa Aesar Chemical Co. Ltd, Tianjin, People's Republic of China. Doxorubicin (DOX) was purchased from Adamas Reagent Ltd, Shanghai, People's Republic of China. All the other chemical reagents were of analytical grade and were used directly without further purification. Deionized (DI) water was used throughout.

\section{Synthesis of $\mathrm{Co}_{3} \mathrm{O}_{4}$ nanoparticles (CoNPs)} The hydrophobic CoNPs were obtained by the solvothermal method using $\mathrm{Co}(\mathrm{acac})_{2}$ and $\mathrm{OA}$ as precursor. ${ }^{33} \mathrm{Co}(\mathrm{acac})_{2}$ was dissolved in $3.6 \mathrm{~mL} \mathrm{OA}, 3.6 \mathrm{~mL} \mathrm{OM}$, and $30 \mathrm{~mL}$ ODE with a final concentration of $350 \mathrm{mM}$ into a $100 \mathrm{~mL}$ three-necked flask. Under the vacuum, the mixture was heated to $100^{\circ} \mathrm{C}$ to form a clear solution. The solution was slowly heated to $200^{\circ} \mathrm{C}$ and maintained for 30 minutes under the Argon atmosphere and then heated to $295^{\circ} \mathrm{C}$ for 240 minutes. After the solution was cooled naturally, the CoNPs were separated via centrifugation $(10,000 \mathrm{rpm})$ and washed with ethanol/ cyclohexane $(1: 1 \mathrm{v} / \mathrm{v})$ three times. The product was stored under room temperature in cyclohexane.

\section{Hydrophilic transfer of CoNPs}

Ligand-free CoNPs were obtained by a reported ligandexchange method. ${ }^{34}$ Typically, $5 \mathrm{~mL}$ CoNPs dispersion in cyclohexane $\left(\sim 5 \mathrm{mg} \mathrm{mL}^{-1}\right)$ was combined with $5 \mathrm{~mL} \mathrm{CH}_{2} \mathrm{Cl}_{2}$ solution of $\mathrm{NOBF}_{4}(0.01 \mathrm{M})$ at room temperature. Then, the nanomaterials were washed with dichloromethane, ethanol, and DI water several times. The CoNPs were coated with BSA by a reported layer-by-layer (LBL) method to achieve hydrophilic transfer. ${ }^{35}$ In a typical experiment, ligand-free CoNPs were then dispersed in a saturated solution of CA as the host reagent, being ultrasonically treated for 10 minutes at room temperature. The resultant mixture was separated via centrifugation and washed several times with DI water to remove the excess CA. In total, $5 \mathrm{~mL}$ CoNPs-CA $\left(1 \mathrm{mg} \mathrm{mL}^{-1}\right)$ was dropped into $10 \mathrm{~mL}$ PAH solution $\left(2 \mathrm{mg} \mathrm{mL}^{-1}\right)$ under ultrasonication for 30 minutes. After stirring for 4 hours, a CoNPs-PAH solution was obtained. Second, the above
CoNPs-PAH solution was dropped into $10 \mathrm{~mL}$ PAA solution $\left(2 \mathrm{mg} \mathrm{mL}^{-1}\right)$ under repeat ultrasonication, stirring, and purification. After adjusting the $\mathrm{pH}$ to 7.4, $50 \mathrm{mg}$ EDC was added into this solution and stirred overnight. Lastly, a solution of BSA ( $5 \mathrm{mg} \mathrm{mL}^{-1}$ ) was added into the CoNPs-PAH-PAA solution, which was ultrasonicated for another 30 minutes. After $50 \mathrm{mg}$ EDC and $50 \mathrm{mg}$ NHS added and $\mathrm{pH}$ adjusted to 7.4 , the mixture was stirred overnight and then purified to obtain CoNPs-BSA. The product was stored under $4^{\circ} \mathrm{C}$ in PBS solution.

\section{Characterization}

The sizes and morphologies of CoNPs were determined using a Tecnai $\mathrm{G}^{2} \mathrm{~F} 30$ transmission electron microscope (TEM) under $300 \mathrm{kV}$ accelerating. Samples of the CoNPs were dispersed in cyclohexane, dropping on the surface of a copper grid. High-resolution lattice image was also obtained during the TEM determination. Powder X-ray diffraction (XRD) measurement was carried out using a Brucker D8 advance $\mathrm{X}$-ray diffractometer from $10^{\circ}$ to $90^{\circ}(\mathrm{Cu} \mathrm{K} \alpha$ radiation, $\lambda=1.54 \AA$ ). Ultraviolet-visible-near infrared (UV-vis-NIR) absorption spectra were obtained on a Shimadzu UV-3600 ultraviolet-visible-near-infrared spectrophotometer. Fluorescence spectra were carried out on Hitachi F-7000 fluorescence spectrophotometer. Dynamic light scattering and zeta potential experiments were carried out on an ALV-5000 spectrometer goniometer equipped with an ALV/LSE-5004 light scattering electronic and multiple tau digital correlator and a JDS Uniphase He-Ne laser $(632.8 \mathrm{~nm})$ with an output power of $22 \mathrm{~mW}$. The size distribution was measured at $25^{\circ} \mathrm{C}$ with a detection angle of $90^{\circ}$. Inductively coupled plasma mass spectroscopy (ICP-MS) analysis was performed on Agilent 7500ce ICP-MS.

\section{Photothermal properties}

Photothermal imaging system was designed as described in previously published literature. ${ }^{36}$ To investigate the photothermal effect, CoNPs-BSA was poured in specimen bottles (total volume of $2.0 \mathrm{~mL}$ ), irradiated by continuouswave diode NIR laser with a center wavelength of $808 \mathrm{~nm}$ and an output power of $1 \mathrm{~W} \mathrm{~cm}^{-2}$. The temperature was measured by a digital thermometer with a thermocouple probe every 0.133 second. The photothermal images were obtained using FLIR E40 equipment running on FLIR tools systems.

\section{Relaxivities measurement and MRI in vitro}

The MRI signal intensity of CoNPs-BSA in tubes was ascertained by the average intensity in the defined regions of 
interests. Relaxivities were determined using a $3 \mathrm{~T}$ siemens Magnetom Trio running on Siemens' Syngo software version B 15 (Siemens Medical Systems, Shanghai, People's Republic of China), in conjunction with an 8 array Loop coil (Siemens Medical Systems). The values of longitudinal relaxivity $\left(r_{1}\right)$ were calculated through the curve fitting of longitudinal relaxation time $\left(\mathrm{s}^{-1}\right)$ vs the concentration of Co $(\mathrm{mM})$. The slope of the line provides the molar $r_{1}$. The transversal relaxivity $\left(\mathrm{r}_{2}\right)$ was determined using the same method.

In vitro $T_{1}$-weighted $M R$ images were obtained using the same equipment as relaxivity determination. CoNPs-BSA solutions in various conditions were placed in a $1.5 \mathrm{~mL}$ tube. The following parameters were adopted: a spin-echo sequence: repetition time $(\mathrm{TR})=350 \mathrm{~ms}$, echo time $(\mathrm{TE})=14 \mathrm{~ms}$, flip angle $=90^{\circ}$, slice thickness $=1.5 \mathrm{~mm}$, FOV read $=150 \mathrm{~mm}$, and case resolution $=256$. $\mathrm{T}_{2}$-weighted $\mathrm{MRI}$ in vivo was obtained with the following parameters: $\mathrm{TR}=3,000 \mathrm{~ms}$, $\mathrm{TE}=20.3 \mathrm{~ms}$, flip angle $=90^{\circ}$, slice thickness $=2.0 \mathrm{~mm}, \mathrm{FOV}$ $\mathrm{read}=200 \mathrm{~mm}$, and case resolution $=256$.

\section{Degradation study}

To investigate the degradation effect, $1 \mathrm{~mL}$ CoNPs-BSA solution $\left(0.25 \mathrm{mg} \mathrm{mL}^{-1}\right)$ was mixed with $1 \mathrm{~mL} \mathrm{AA}$ solution with various concentrations under various $\mathrm{pH}$ conditions. At various time points, the mixed solution was dropped on the surface of a copper grid and the excess liquid was quickly absorbed using a filter paper. Moreover, the UV-vis-NIR spectra were also taken at the same time point. The degradation efficiency of CoNPs-DOX was also determined using the same method.

\section{Drug delivery and controlled release}

In total, $2 \mathrm{mg}$ CoNPs-BSA was dissolved in $2 \mathrm{~mL}$ DOX solution ( $\left.1 \mathrm{mg} \mathrm{mL}^{-1}\right)$, stirred for 10 hours and separated via centrifugation and washed with DI water for three times to remove the excess DOX. The obtained DOX-loaded CoNPs (CoNPs-DOX) were dissolved in DI water $\left(1 \mathrm{mg} \mathrm{mL}^{-1}\right)$ and stored under $4^{\circ} \mathrm{C}$. The drug delivery amount of each group was verified by UV-vis-NIR spectra. To study the controlled release properties of each group, $1 \mathrm{~mL}$ AA solution ( $5 \mathrm{mg} \mathrm{mL}^{-1}$ ) was added into the above solution. The mixture was centrifuged to get the supernatant, and UV-vis-NIR spectra of the obtained supernatant at various time points were then determined.

\section{Photothermal imaging in vivo}

All the animal procedures were performed in accordance with the Guidelines for Care and Use of Laboratory Animals of Beijing Vital River Laboratory Animal Technology Co., Ltd,
Beijing, People's Republic of China and approved by the Animal Ethics Committee of the Vital River Institutional Animal Care and Use Committee (VR IACUC). Nude mice bearing HCT116 tumors were used for in vivo photothermal imaging experiment. Before imaging, the mouse was anesthetized and set on a mount designed by our own group. ${ }^{36}$ Photothermal imaging was performed before and after intravenous injection of CoNPs-DOX (10 mg per kg body weight of mouse) with $808 \mathrm{~nm}$ irradiated at $1 \mathrm{~W} \mathrm{~cm}^{-2}$ at various time points. Photothermal images were acquired and analyzed using the FLIR software.

\section{Photothermal therapy in vivo}

When the tumor size reached $\sim 200 \mathrm{~mm}^{3}$, nude mice bearing HCT116 tumors were randomly divided into five groups for photothermal therapy experiments. In the test group, mice were intravenously injected with DOX-loaded CoNPs (CoNPs-DOX, $10 \mathrm{mg}$ per kg body weight of mouse), exposed to an $808 \mathrm{~nm}$ laser, and then intravenously injected with AA (CoNPs-DOX + laser + AA). For comparison, mice without AA injection (CoNPs-DOX + laser), mice with CoNPs injection and laser irradiation (CoNPs + laser), and mice with CoNPs injection and no laser irradiation (CoNPs) were chosen as control groups. Mice without any treatment were chosen as the blank group. A caliper was used to measure the sizes of tumor every 3 or 4 days after photothermal treatment.

\section{Cell culture}

HCT116 (human colon carcinoma cells) and CCC-HEL-1 (human diploid hepatocyte cells) cell lines were provided by the Institute of Basic Medical Sciences, Chinese Academy of Medical Sciences. Cells were grown in DMEM supplemented with $10 \% \mathrm{FBS}$ and $1 \%$ penicillin-streptomycin at $37^{\circ} \mathrm{C}$ with $5 \% \mathrm{CO}_{2}$.

\section{MTT study}

Cells $\left(90 \mu \mathrm{L}\right.$ well $\left.{ }^{-1}, 10^{5} \mathrm{~mL}^{-1}\right)$ were put into 96-well cell-culture plate and then cultured at $37^{\circ} \mathrm{C}$ under $5 \% \mathrm{CO}_{2}$ for 24 hours. CoNPs-BSA $\left(10 \mu \mathrm{L}\right.$ well $\left.{ }^{-1}\right)$ at various concentration were added to the wells of the experimental groups, and $10 \mu \mathrm{L}$ PBS in the control group. The cells were cultured at $37^{\circ} \mathrm{C}$ for 24 and 48 hours, respectively. Then, 3-(4,5-dimethyl-2-thiazolyl)-2,5-diphenyl-2-H-tetrazolium bromide (MTT, $10 \mu \mathrm{L}, 5 \mathrm{mg} \mathrm{mL}^{-1}$ ) was added to the wells and incubated at $37^{\circ} \mathrm{C}$ for 4 hours. After the addition of dimethyl sulfoxide $\left(100 \mu \mathrm{L}\right.$ well $\left.{ }^{-1}\right)$, the assay plate was allowed to stand at room temperature for 30 minutes. Tecan Infinite M200 monochromator-based multifunction microplate reader was used to measure the OD570 (absolute value) of each well with background subtraction. The following formula was 
used to calculate the viability of cell growth: Cell viability $(\%)=$ (mean of absolute value of treatment group)/(mean absolute value of control) $\times 100 \%$.

\section{Hematology studies}

Blood was harvested from mice intravenously injected with CoNPs-BSA (20 mg per kg body weight of mouse) and from mice receiving PBS injection (control), 1 day, 7 days, 30 days, and 60 days postinjection. Blood was collected from the orbital sinus by quickly removing the eye ball from the socket with a pair of tissue forceps. Five important hepatic indicators (alanine aminotransferase [ALT], aspartate aminotransferase [AST], total bilirubin [TBIL], albumin [ALB], and total protein [TP]), and one indicator for kidney functions (creatinine [CRE]) were measured. Blood smears were prepared by placing a drop of blood on one end of a slide, and using another slide to disperse the blood along the length of the slide. The slide was left to air dry, after which the blood was stained with H\&E. Upon completion of the blood collection, mice were sacrificed.

\section{H\&E-stained tissue sections}

The heart, liver, spleen, lung, and kidney were harvested from mice intravenously injected with CoNPs-BSA (20 mg per kg body weight of mouse) and from mice receiving no injection (control), 1 day, 7 days, 30 days, and 60 days postinjection. The tissues were fixed in paraformaldehyde, embedded in paraffin, sectioned, and stained with $\mathrm{H} \& \mathrm{E}$. The histological sections were observed under an optical microscope.

\section{Results and discussion}

\section{Synthesis and characteristic of CoNPs-BSA}

The CoNPs were prepared by solvothermal method in highboiling point solvent. TEM image of CoNPs suggested that the
A

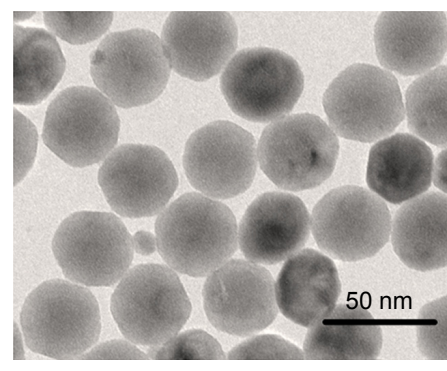

D

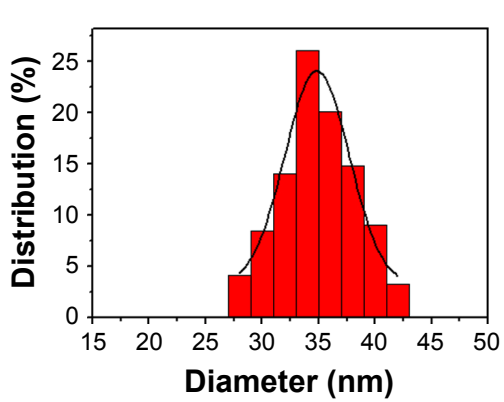

G

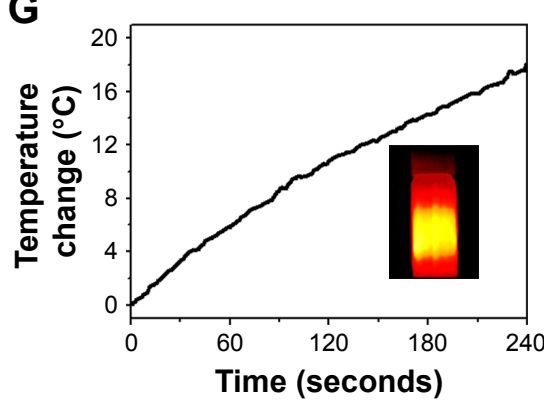

B

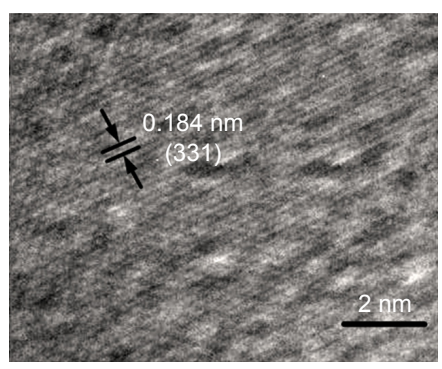

$\mathrm{E}$

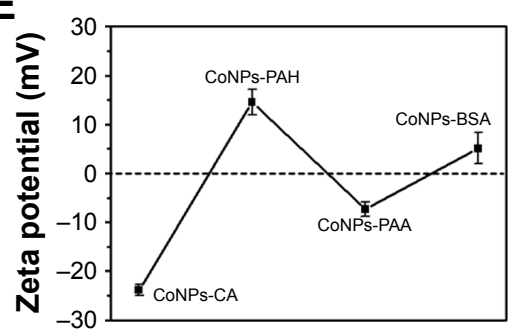

H

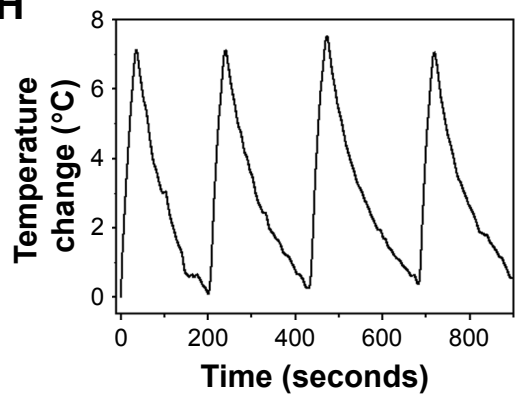

C

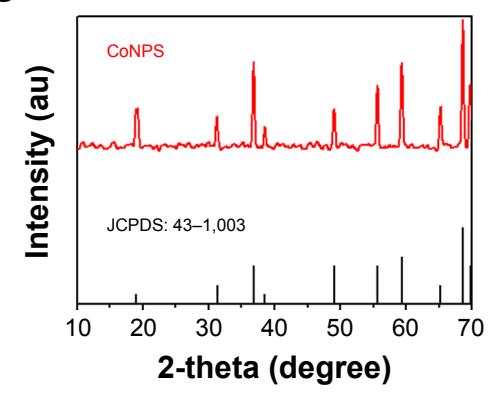

$\mathbf{F}$

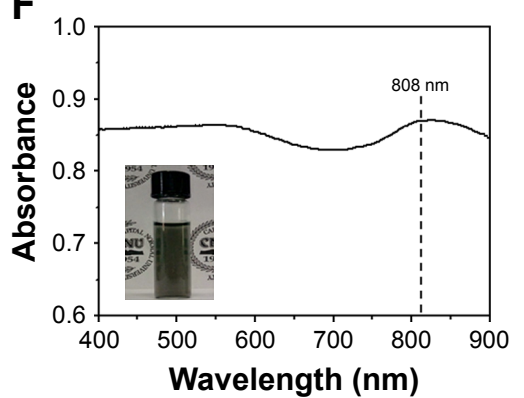

I

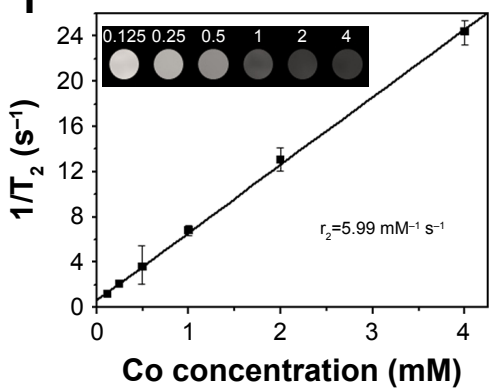

Figure I The chemical and physical characterization of CoNPs-BSA.

Notes: TEM image (A), HR-TEM image (B), XRD patterns (C), and size distribution (D) of as prepared CoNPs. (E) Zeta potential of CoNPs within the layer-by-layer assembly process. (F) UV-vis-NIR spectra of CoNPs-BSA. (G) Photothermal temperature change curve of CoNPs-BSA. (H) Temperature variations of CoNPs-BSA under the continuous irradiations of $808 \mathrm{~nm}$ laser at $\mathrm{I} .0 \mathrm{~W} \mathrm{~cm} \mathrm{cor}^{-2}$ four cycles. (I) Transversal relaxation time $\left(\mathrm{I} / \mathrm{T}_{2}\right)$ vs concentration of $\mathrm{Co}_{3} \mathrm{O}_{4}-\mathrm{BSA}$.

Abbreviations: CoNPs, $\mathrm{Co}_{3} \mathrm{O}_{4}$ nanoparticles; HR-TEM, high-resolution transmission electron microscopy; TEM, transmission electron microscopy; UV-vis-NIR, ultravioletvisible-near infrared; $\mathrm{XRD}, \mathrm{X}$-ray diffraction. 
CoNPs were uniform spheres with high crystalization and size distribution of $35.74 \mathrm{~nm}$ (Figure 1A, B, and D). XRD patterns of CoNPs were corresponded to the standard face-centered cubic (FCC) $\mathrm{Co}_{3} \mathrm{O}_{4}$ (JCPDS: 43-1003), demonstrating that the as-prepared CoNPs were $\mathrm{FCC} \mathrm{Co}_{3} \mathrm{O}_{4}$ (Figure 1C). To improve the biocompatibility and hydrophilicity of as-prepared CoNPs, CA, PAA, and PAH were sequentially coated onto CoNPs surfaces by a typical LBL method. ${ }^{37}$ Then, BSA was further conjugated by the formation of amide bonds. Within the modification process, the zeta potential increased from $-23.1 \mathrm{mV}$ (CoNPs-CA) to $16.3 \mathrm{mV}$ (CoNPs-PAH), and then decreased to $-8.7 \mathrm{mV}$ (CoNPs-PAA) and finally increased to $4.17 \mathrm{mV}$ after BSA modification (CoNPs-BSA) (Figure 1E). The hydrodiameter of CoNPs in the LBL modification process also supported the same findings (Figure S1). The CoNPs-BSA possessed high stability in water, PBS, and saline (Figure S2). These results confirmed the successful preparation and modification of CoNPs-BSA with controllable structure and high stability for further study and application.

By UV-vis-NIR determination, it was found that CoNPsBSA have strong absorbance in the range of 600-900 nm, which may contribute to the surface plasmonic resonance property of semiconductors (Figure 1F). The molar extinction coefficient $(\varepsilon)$ was further calculated for comparison by using Equation 1:

$$
\varepsilon=\frac{A V_{N P S} \rho N_{A}}{L C}
$$

The $\varepsilon$ of CoNPs was calculated to be $5.74 \times 10^{7} \mathrm{M}^{-1} \mathrm{~cm}^{-1}$, which adapted it as a potential photothermal conversion agent. We then determined the temperature change of the CoNPs suspension under $808 \mathrm{~nm}$ laser irradiation to confirm the photothermal conversion effect (Figure 1G). The result suggested that CoNPs-BSA has a high temperature change of $18.1^{\circ} \mathrm{C}$ within 5 minutes when excited by $808 \mathrm{~nm}$ laser, which may be a cooperative result of the high $\varepsilon$ and photothermal conversion efficiency $(\eta)$ of CoNPs-BSA ( $\eta=46.19 \%)$ (Figure S3). It was also found that the CoNPs-BSA was a robust photothermal conversion agent after receiving several heating-cooling cycles (Figure $1 \mathrm{H}$ ). In addition of the properties related with photothermal performance, the magnetic resonance property was also taken into consideration by determining transversal relaxivity $\left(\mathrm{r}_{2}\right)$ under $3 \mathrm{~T}$ clinical magnetic field intensity (Figure 1I). It was found that CoNPsBSA also has a high transversal relaxivity of $5.99 \mathrm{mM}^{-1} \mathrm{~s}^{-1}$, which promised their potential in the $\mathrm{T}_{2}$-weighted MRI of tumor. Furthermore, the longitudinal relaxivity $\left(\mathrm{r}_{1}\right)$ was determined to be $0.17 \mathrm{mM}^{-1} \mathrm{~s}^{-1}$. The $\mathrm{r}_{2} / \mathrm{r}_{1}$ was calculated to be 35.24, which suggested that the CoNPs-BSA was a typical $\mathrm{T}_{2}$-weighted MRI contrast agent. By quantificationally studying the performance of CoNPs-BSA in photothermal conversion and magnetic resonance, it was clear that CoNPs-BSA can be an optimal nanomaterial for further cancer theranostics study.

\section{The degradation and drug delivery/ release properties of CoNPs-BSA}

To further illustrate the feasibility of CoNPs-BSA for cancer theranostics, detailed study was taken focusing on the degradation and drug delivery/release both in vitro and in vivo. AA, a nontoxic water-soluble vitamin with strong reducibility, was suitable for the redox degradation of CoNPs-BSA in vivo. By mixing CoNPs-BSA with AA, it was found that the absorbance at $808 \mathrm{~nm}$ decreased rapidly, which can serve as the indicator to monitor the degradation process (Figure S4). The influence of AA concentration on CoNPs-BSA degradation efficiency was further studied by UV-vis-NIR spectra. The results suggested that the CoNPs-BSA can be degraded in neutral solution containing various AA concentrations (Figure 2A). The proposed degradation mechanism can be implied as the following chemical equation:

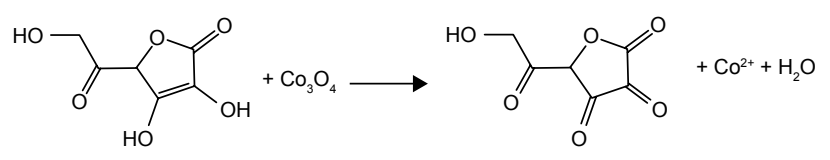

Importantly, the degradation efficiency was positively related with both AA concentration and reaction time. In addition, the $\mathrm{pH}$ condition also has influence on the degradation efficiency (Figure 2B). Under the acidic condition ( $\mathrm{pH}=5.5)$, the CoNPs-BSA was degraded much more rapidly and efficiently, compared with those in neutral condition $(\mathrm{pH}=7.5)$. Importantly, in the absence of AA, the CoNPsBSA showed high stability in both acidic and neutral condition, which may be a result of the protection of coated BSA by residual amino protonation. ${ }^{16}$ As a result of the highly efficient degradation, the contrast switch from $\mathrm{T}_{2}$ - to $\mathrm{T}_{1}$-weighted MRI was clearly identified by the continuous decrease in longitudinal relaxation time $\left(\mathrm{T}_{1}\right)$ and increase in transversal relaxation time $\left(\mathrm{T}_{2}\right)$ (Figure $2 \mathrm{C}$ ). This phenomenon can be explained as the degradation of CoNPs and production of paramagnetic $\mathrm{Co}^{2+}$ with numerous unpaired electrons. To further confirm the degradation of CoNPs-BSA in presence of AA, TEM images of the degradation products were taken, which reveal that CoNPs-BSA are no longer nanospheres 
A

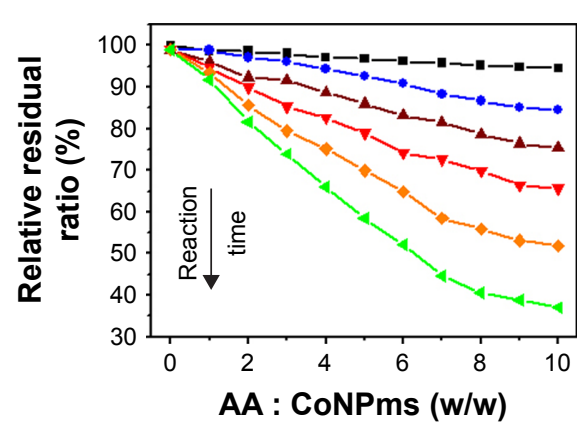

D

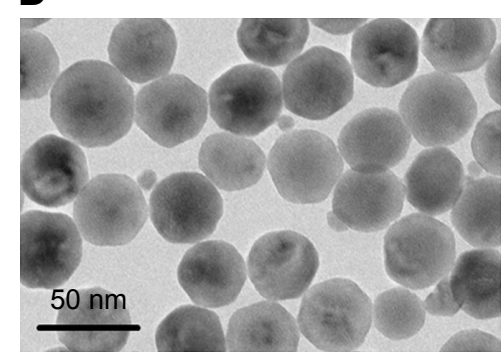

G

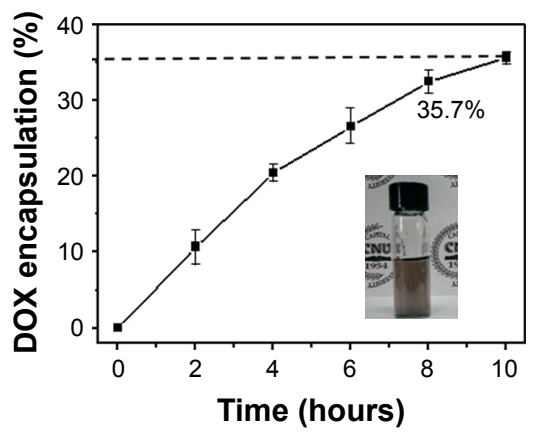

B

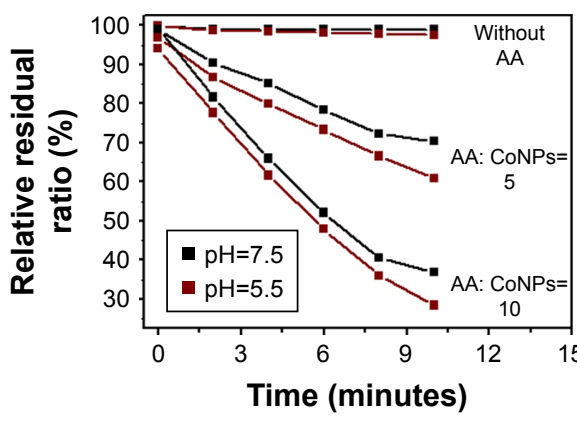

E

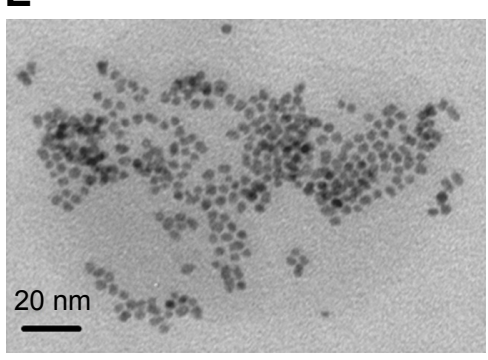

H

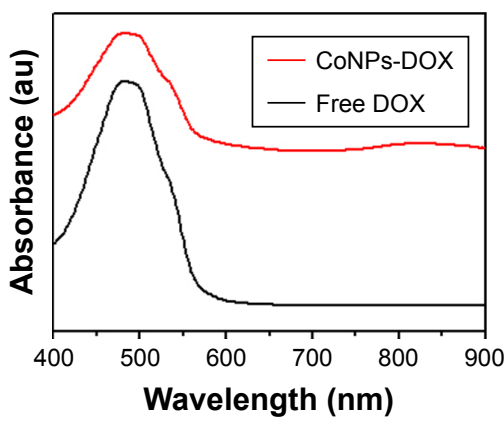

I
C

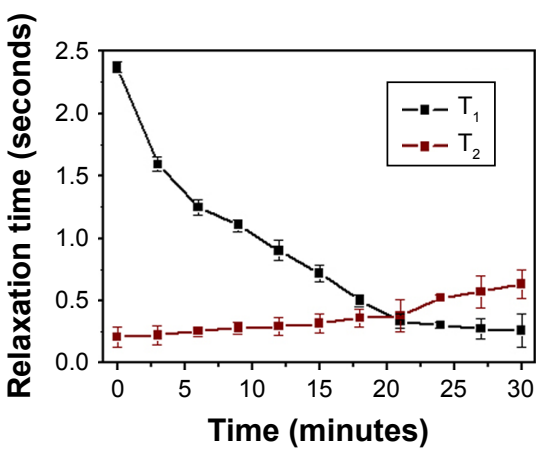

F
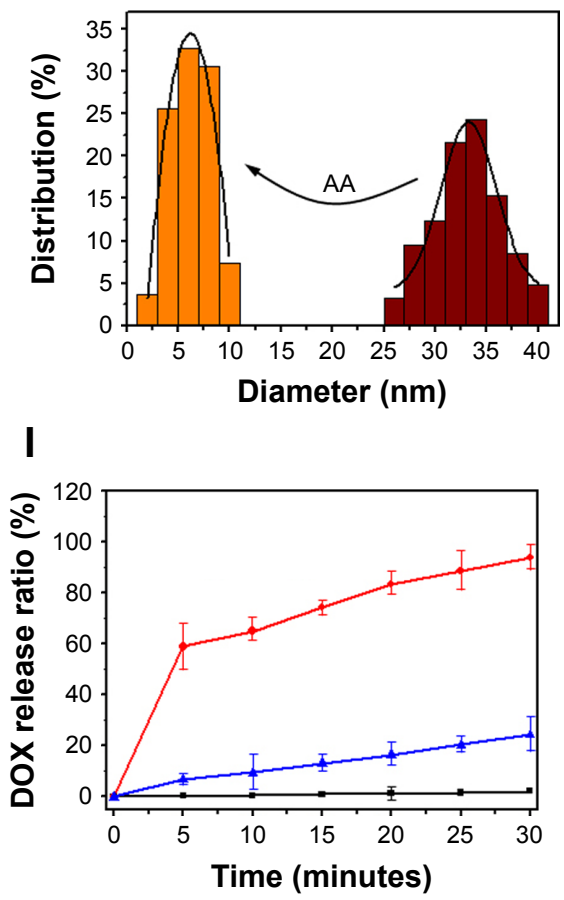

$\rightarrow-\mathrm{AA}(-)$ laser $(-)$

$\rightarrow$ AA (-) laser (+)

$\rightarrow$ AA $(+)$ laser $(-)$

Figure 2 Degradation and drug delivery capacities of CoNPs-BSA.

Notes: (A) The degradation ratio of CoNPs-DOX within 10 minutes in the presence of AA with various ratio. (B) The influence of acidic condition on the degradation efficiency of CoNPs-DOX by AA. (C) The longitudinal $\left(T_{1}\right)$ and transversal $\left(T_{2}\right)$ relaxation time of CoNPs-DOX suspension within 30 minutes after AA added. TEM images of CoNPs-DOX in the absence (D) and presence (E) of AA within 10 minutes. (F) Size distribution of CoNPs-DOX in the absence and presence of AA within 10 minutes. (G) Drug encapsulation efficiency of CoNPs-BSA. (H) UV-vis-NIR spectra of free DOX and CoNPs-DOX. (I) DOX release ratio from CoNPs-DOX without AA addition and laser irradiation $(\mathrm{AA}(-)$ laser $(-))$, with only laser irradiation $(\mathrm{AA}(-)$ laser $(+))$, and with only $\mathrm{AA}$ addition $(\mathrm{AA}(+)$ laser $(-))$.

Abbreviations: AA, ascorbic acid; $\mathrm{CoNPs}, \mathrm{Co}_{3} \mathrm{O}_{4}$ nanoparticles; DOX, doxorubicin; TEM, transmission electron microscopy; UV-vis-NIR, ultraviolet-visible-near infrared.

but smaller irregular particles with the diameter of $\sim 3 \mathrm{~nm}$ (Figure 2D-F). The above results indicate that the CoNPsBSA can be degraded by AA via a reduction process.

Further effort was made on the drug delivery and controlled release properties of CoNPs-BSA. As a typical drug in clinical cancer therapy, DOX was chosen as the drug model for further study. ${ }^{38-41}$ Since DOX has a strong absorbance peak in the visible range and excellent linear range was found between absorbance intensity and drug concentration,
UV-vis-NIR spectra were used for the identification and quantification of drug delivery efficiency. It was found that the CoNPs-BSA can effectively load DOX, with a high encapsulation efficiency of $35.7 \%$ (Figure 2G). The absorbance peak was also observable in the UV-vis-NIR spectra of CoNPs-DOX, which strongly evidences the successful drug loading (Figure 2H).

As an essential factor for the evaluation of a drug nanocarrier, the controlled drug release performance of CoNPs-DOX 
was also studied carefully. Previous reports pointed out that the drug release can be triggered by localized photothermal. Hence, we studied the photothermal triggered drug release first. The result illustrated that only small amount of DOX was released from CoNPs-DOX within 30 minutes ( 20\%), which may be a result of limited temperature change. To achieve better drug release efficacy, a much more effective trigger was required. As the CoNPs-DOX degraded in AA presence, the surface layer of CoNPs was decomposed, weakening the electrostatic interaction between DOX and CoNPs, further resulting in the release of DOX. Therefore, the addition of AA can be an important trigger for drug release. The CoNPsDOX showed high DOX release amount $(>90 \%)$ in the presence of AA than in the AA absence condition (Figure 2I). It is important to find that the loaded drug can be burningly released within the first 5 minutes after AA added. With low AA concentration in the body of mammal, AA-triggered drug release was in the requirement of supernumerary AA uptake. Owing to the low drug release amount within photothermal process, the use of AA trigger allowed a programmed cancer therapy strategy that activated chemotherapy after photothermal therapy, achieving highly efficient long-term therapy. All the facts, including highly efficient drug delivery and AAtriggered drug release, suggested that the CoNPs-DOX can be served as a promising drug delivery vehicle for artificially controllable drug release and an important candidate for programmed photothermal-chemo synergistic cancer therapy.

\section{Imaging-guided synergistic therapy in vivo}

As a novel biomaterial for cancer theranostics, the biobehavior of CoNPs-DOX was an essential factor to evaluate its potential in practical application. We primarily studied the biodistribution and metabolism of CoNPs-DOX by ICP-MS, using $\mathrm{Co}$ as indicator. It can be observed from Figure $3 \mathrm{~A}$ and $\mathrm{B}$ that CoNPs-DOX has prolonged blood circulation time and high accumulation amount in the tumor region within 240 minutes after intravenous injection, which was attributed to the enhanced permeability and retention effect. In addition, there is also accumulation in the reticuloendothelial system over times, like liver and spleen. As a result of the above factors, the CoNPs-DOX can accumulate in the tumor region for theranostics. Since CoNPs-DOX can be an outstanding $\mathrm{T}_{2}$-weighted MRI contrast agent and showed $\mathrm{T}_{1}$ contrast enhancement after degradation, we firstly illustrate the feasibility using MRI to monitor CoNPs degradation and drug release in vivo. After intravenous injection of CoNPs-DOX into tumor-bearing mice, $\mathrm{T}_{2}$-weighted MR images were taken at various time points. The images showed obvious retention of CoNPs-DOX in the tumor region (Figure 3C). AA was then injected into mice intravenously 120 minutes after CoNPs-DOX injection and then $\mathrm{T}_{1}$-weighted MR images were taken. It can be found that the tumor and liver site being bright within 30 minutes postinjection corresponded to the biodistribution of AA and also demonstrated the degradation of CoNPs-DOX (Figure 3D and E). The above results confirmed that the $\mathrm{T}_{2}$-weighted MRI can be used for drug delivery monitoring, and the contrast switch was useful for degradation identification as well as drug release.

Apart from the MRI, the synergistic therapeutic efficiency was also evaluated in vivo. The photothermal temperature change of mice in the tumor site was recorded under an $808 \mathrm{~nm}$ laser at a power density of $1 \mathrm{~W} \mathrm{~cm}$. Before intravenous injection, the temperature of tumors was merely changed $\left(<3^{\circ} \mathrm{C}\right.$ within 5 minutes). However, the temperature in tumor site of mice receiving intravenous CoNPs-DOX injection increased over time and reached $45^{\circ} \mathrm{C}$ within 5 minutes (Figure $4 \mathrm{~A}$ and $\mathrm{B}$ ). The temperature changes that occurred at different time points after injection also suggested that CoNPs-DOX was passively targeted and accumulated in the tumor site. According to previous reports, the hyperthermia treatment at a temperature over $42^{\circ} \mathrm{C}$ would result in irreversible injury, by significantly increasing biological reaction rate, irreversible protein denaturation, and DNA damage. Therefore, the injection of CoNPs-DOX, with the assistance of laser, can possess highly efficient tumor ablation. The in vivo AA-triggered drug release can also be confirmed by the luminescence intensity in the tumor regions (Figures $4 \mathrm{C}$ and $\underline{\mathrm{S} 5}$ ). As shown in Figure $4 \mathrm{C}$, the luminescence was much stronger in the tumor harvested from the mice receiving AA injection than those without AA uptake. By Fröster resonance energy transfer between CoNPs and DOX, the luminescence of DOX can be quenched by CoNPs when loaded and would recover after release. The tumor size was then measured to evaluate the therapeutic efficiency. Upon irradiation at $808 \mathrm{~nm}\left(1 \mathrm{~W} \mathrm{~cm}^{-2}\right)$ for 5 minutes, the tumors of the mice in the test group shrank within the first 7 days (Figure 4D). On day 7 , the tumors disappeared, left with only black scars and no obvious swollen parts. It is noteworthy that the tumor size in the test group was significantly different from that of mice receiving synergistic therapy without AA, which highlighted the importance of programmed cancer therapy. This insisted that AA can be an important trigger to release more drugs. Corresponding to the tumor ablation experiment, the body weight of mice also supported the conclusion that mice receiving AA injection had much better therapeutic 
A

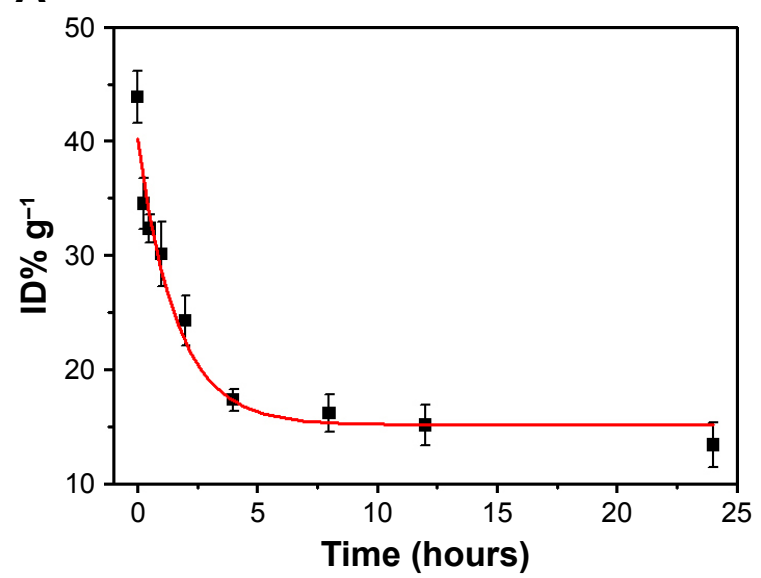

B

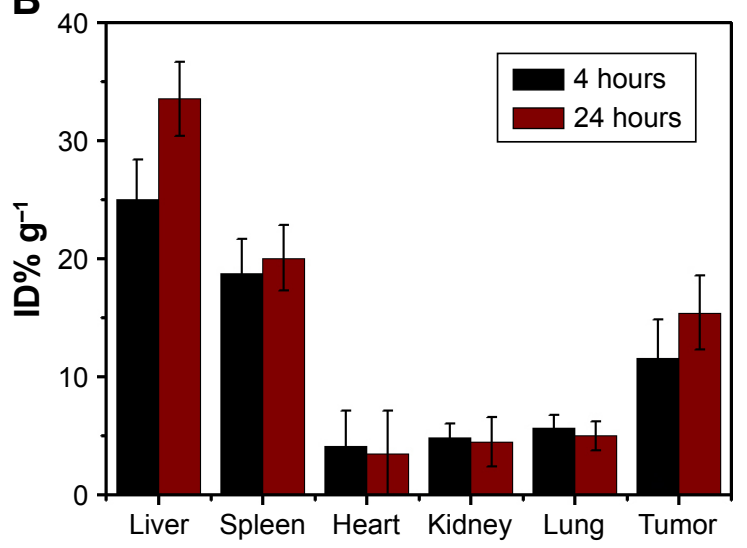

C $\backslash$ ConPs injection (iv)

AA injection (iv)

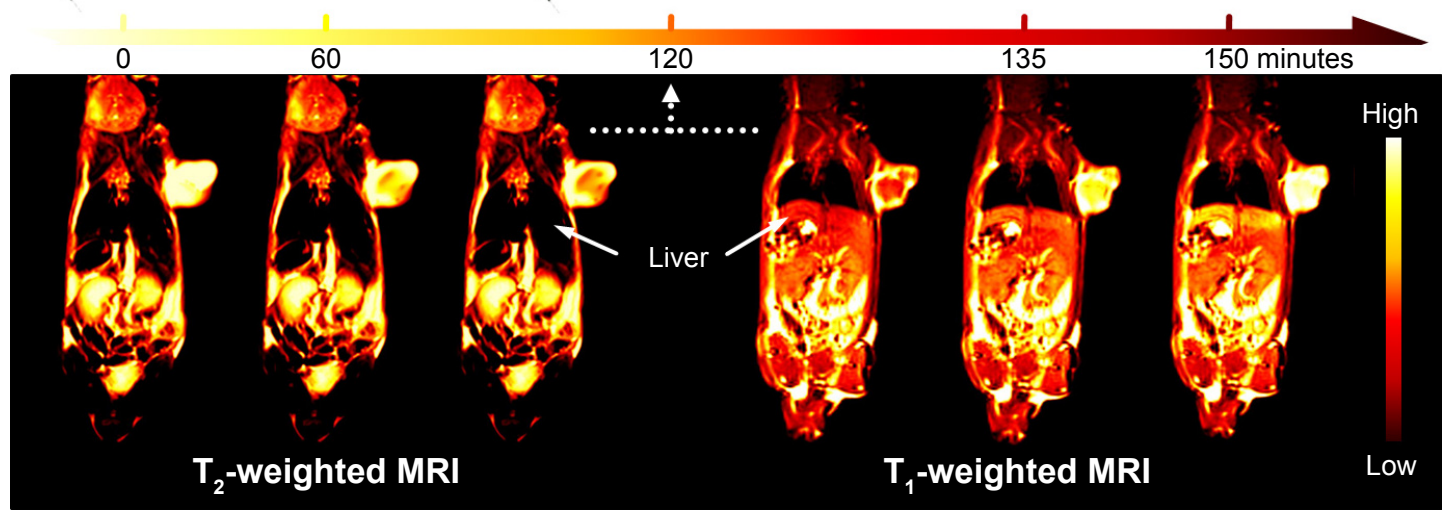

D

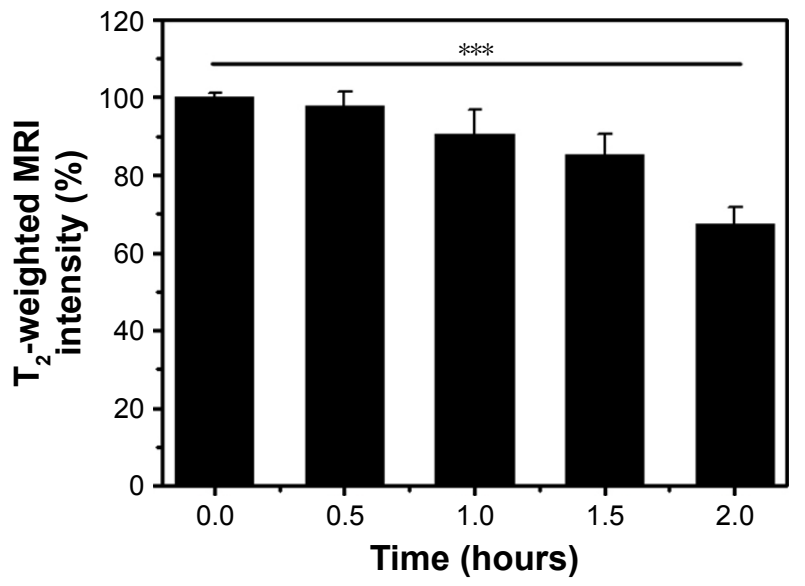

E

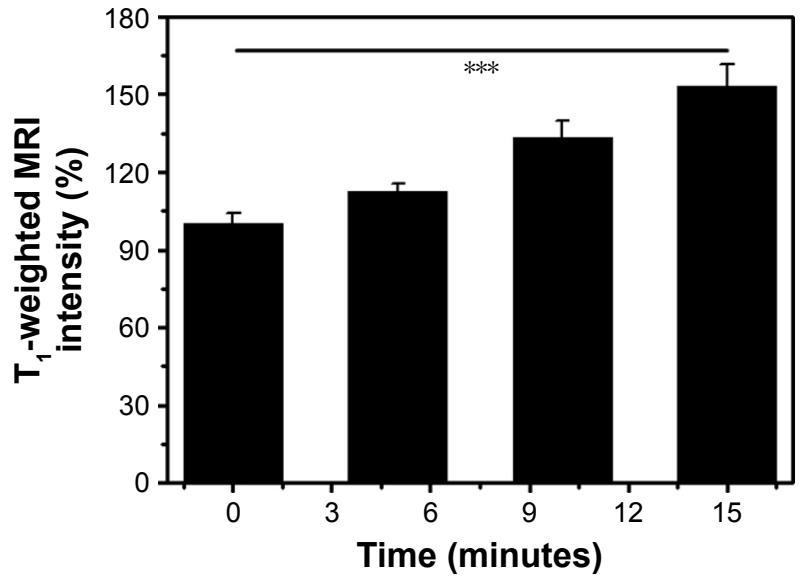

Figure 3 Biobehavior and MRI capacities of CoNPs-DOX in vivo.

Notes: Blood circulation curve $(\mathbf{A})$ and accumulation amount in various organs (B) of CoNPs-DOX after intravenous injection at different time points postinjection (C) $T_{2}$-weighted MRI images of tumor-bearing mice within 120 minutes receiving CoNPs injection and $T_{1}$-weighted MRI images within 30 minutes after $A A$ injection. $\mathrm{T}_{2}$-weighted MRI intensity (D) obtained from the mice receiving CoNPs injection within 120 minutes and $\mathrm{T}_{1}$-weighted MRI intensity (E) in the tumor region after $A A$ injection within 15 minutes. Statistical significance was determined from one-way $t$-tests. $* * * P<0.001$.

Abbreviations: $\mathrm{AA}$, ascorbic acid; $\mathrm{CoNPs}, \mathrm{CO}_{3} \mathrm{O}_{4}$ nanoparticles; DOX, doxorubicin; MRI, magnetic resonance imaging; ID, injected doses.

efficiency than the other groups (Figure 4E). The TEM image of CoNPs-DOX generated from urine of mice receiving AA injection showed that the CoNPs-DOX were degraded to small particles with BSA coating, which can also be a solid evidence of the AA-triggered degradation and drug release (Figure S6). In vivo experiments demonstrated that the CoNPs, loaded with drugs like DOX, could provide a highly effective programmed synergistic cancer therapeutic 
A

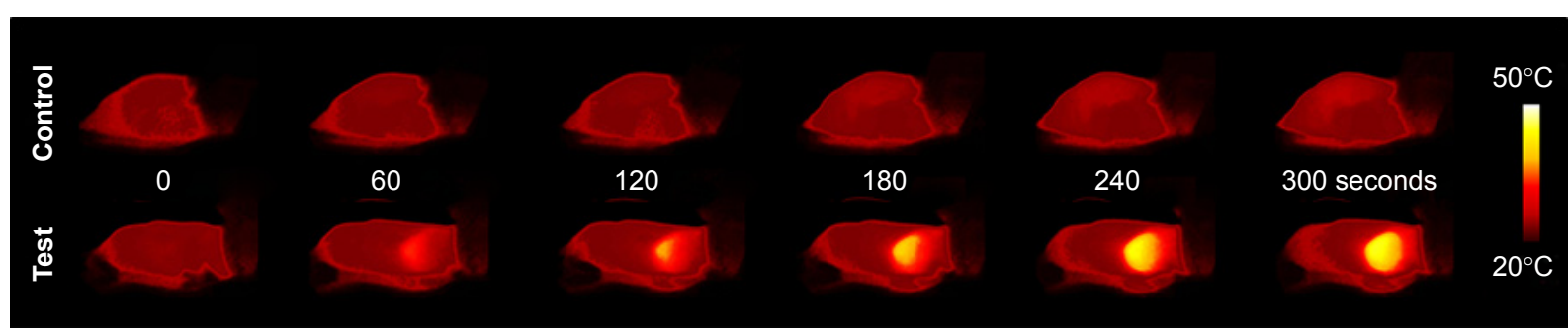

B

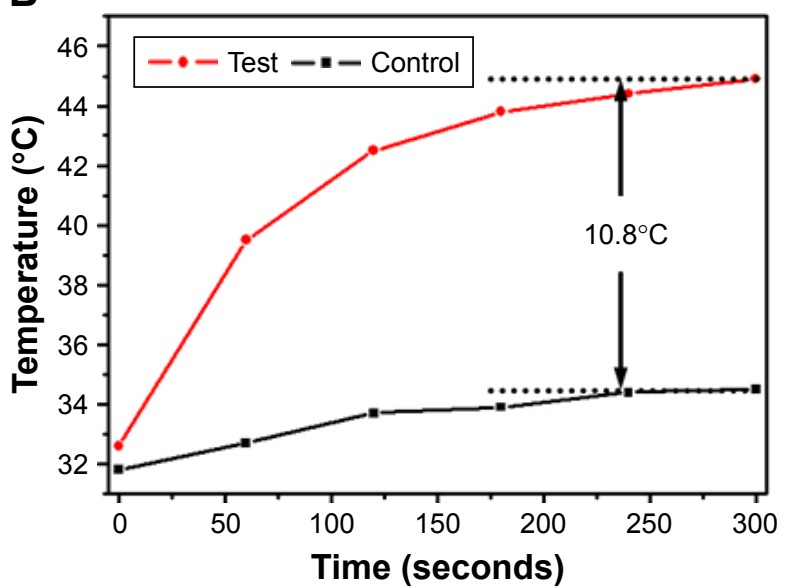

D

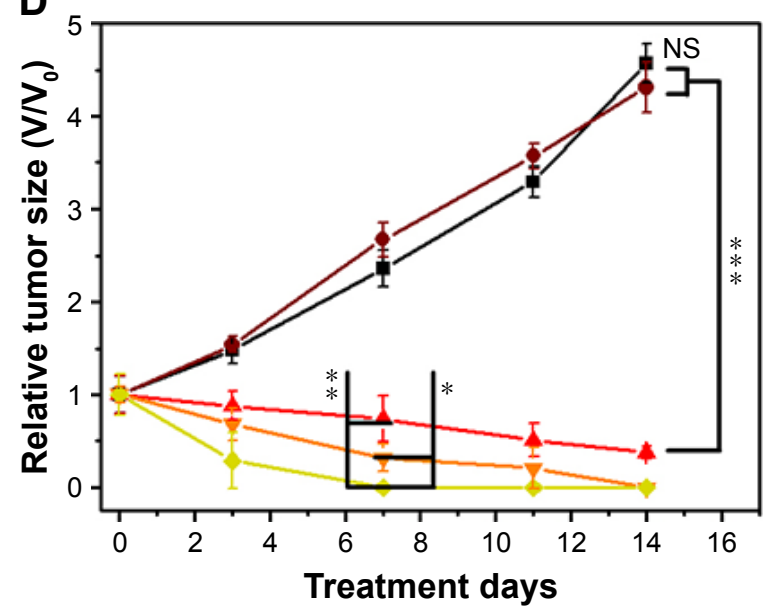

C

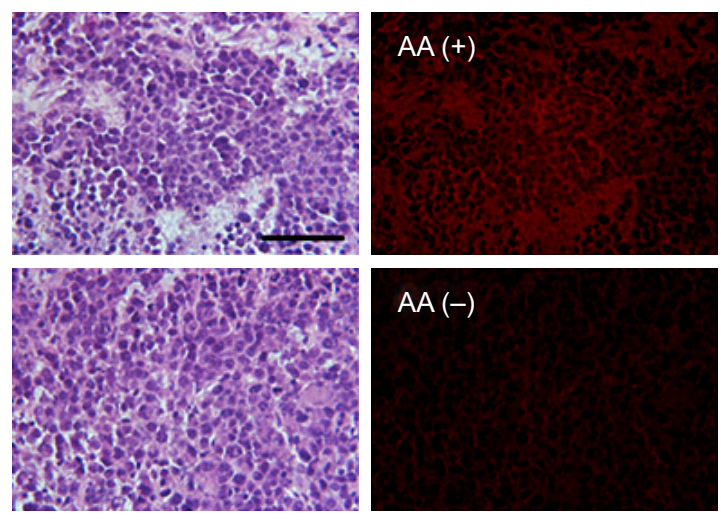

E

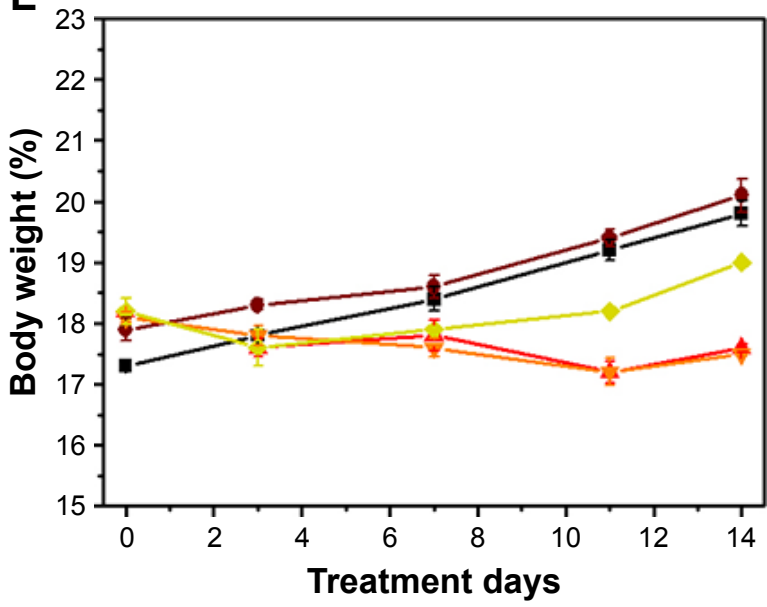

$\longrightarrow$ CoNPs-DOX + laser + AA $\multimap-$ CoNPs-DOX + laser $\simeq$ CoNPs-DOX $\multimap$ CoNPs $\rightarrow$ Blank

Figure 4 Synergistic tumor ablation in vivo.

Notes: Photothermal images $(\mathbf{A})$ and temperature change $(\mathbf{B})$ of tumor-bearing mice receiving CoNPs injection. (C) Tissue section and luminescence images of tumor harvested from mice receiving CoNPs injection and AA injection. Mice receiving no AA injection were used as control. The relative tumor size (D) and body weight (E) of mice in the test group (CoNPs-DOX + laser + AA), control groups (CoNPs-DOX + laser, CoNPs + laser, and CoNPs), and blank group (PBS). The scale bar was $50 \mu \mathrm{m}$. Statistical significance was determined from one-way $t$-tests. $* P<0.05, * * P<0.01$, and $* * * P<0.001$.

Abbreviations: AA, ascorbic acid; CoNPs-DOX, $\mathrm{Co}_{3} \mathrm{O}_{4}$ nanoparticles-doxorubicin; DOX, doxorubicin; NS, not significant.

method by combining photothermal conversion therapy with AA-induced artificially controllable drug release.

\section{Toxicity study}

As the cytotoxicity and biocompatibility were also the important factors to evaluate a biomaterial, the toxicity effect of CoNPs-BSA on both cells and mice was studied. MTT assay showed no significant difference in proliferation of the HCT116 cells in the absence or presence of CoNPs-BSA (0-1.0 $\mathrm{mg} \mathrm{mL}^{-1}$ ) within 24 and 48 hours (Figure 5A). Cellular viabilities were higher than $85 \%$ even at a high dose within 48 hours. Low cytotoxicity results can also be obtained from another MTT assay using human diploid hepatocyte (CCCHEL-1 cells) as a model (Figure 5B). The MTT assay results demonstrated that CoNPs-BSA showed low cytotoxicity. Furthermore, healthy mice were injected intravenously with 
A

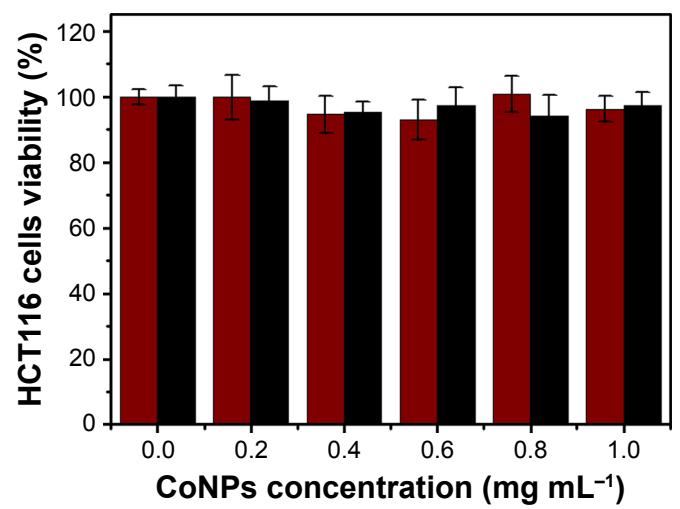

B

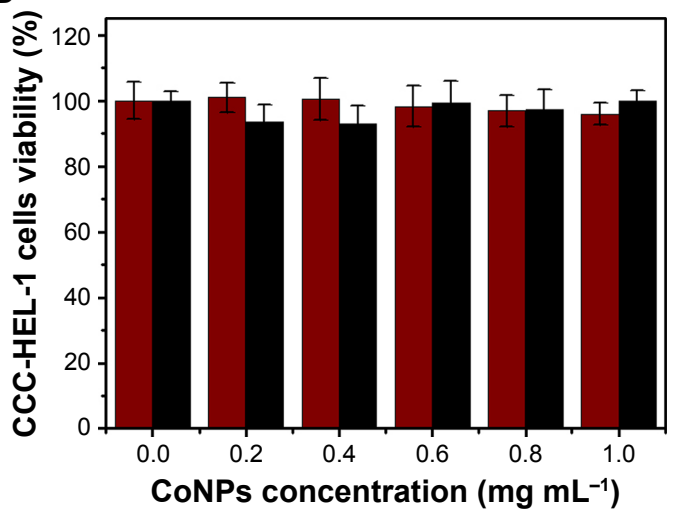

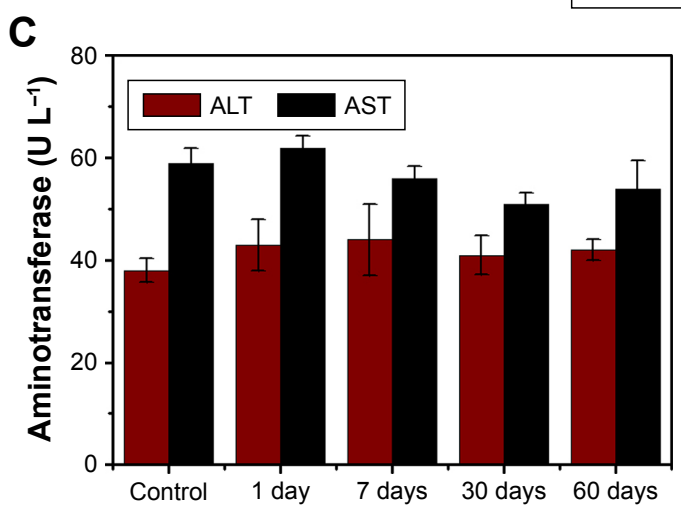

24 hours 48 hours

D

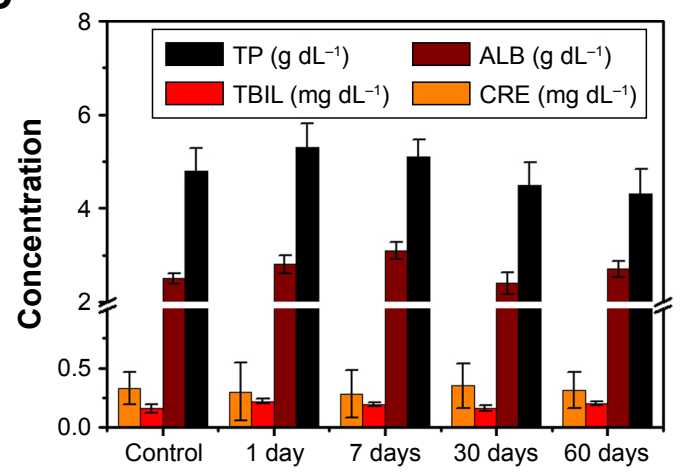

E
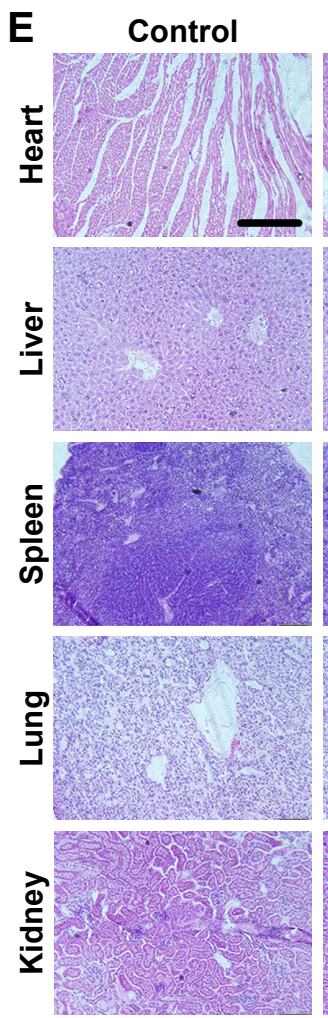

1 day
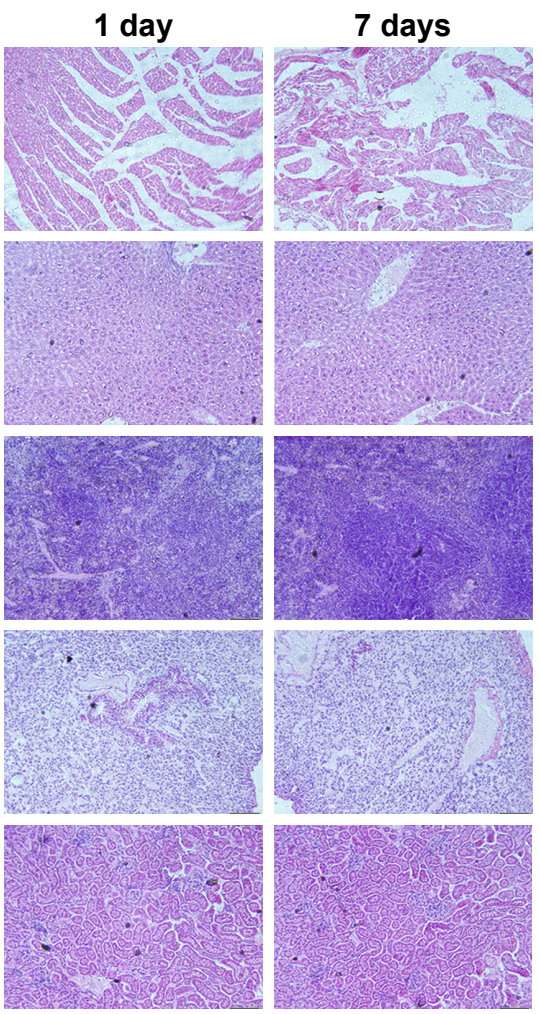
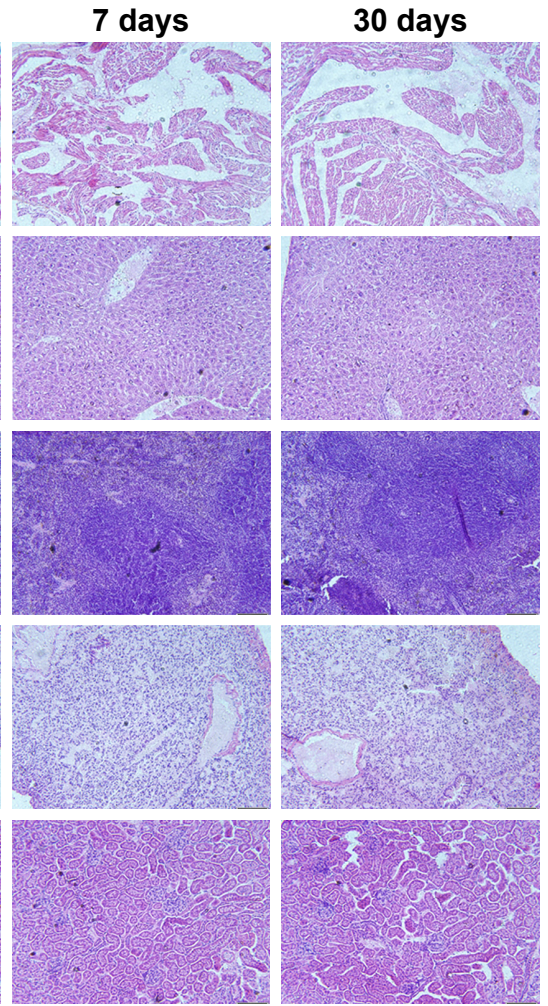

60 days

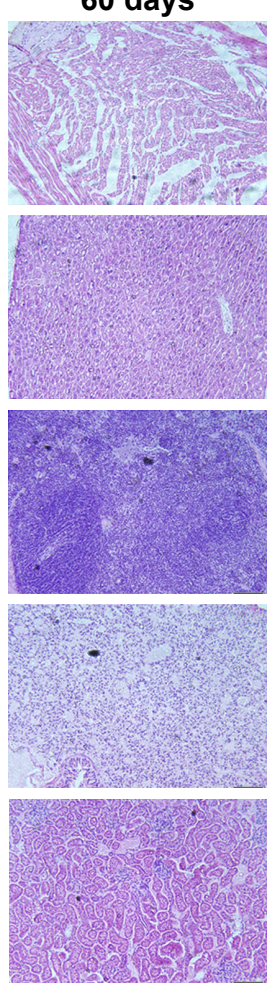

Figure 5 Cytotoxicity and biocompatibility of CoNPs-BSA.

Notes: The viability of HCTII6 cells (A) and CCC-HEL-I cells (B) determined by MTT assay. Two aminotransferase (ALT and AST) (C), typical liver/kidney function indicators (TP, ALB, TBIL, and CRE) (D), and H\&E stained tissue sections (E) of mice receiving CoNPs-BSA injection within 60 days postinjection. Mice receiving PBS injection was used as the control group. The scale bar was $50 \mu \mathrm{m}$.

Abbreviations: ALB, albumin; ALT, alanine aminotransferase; AST, aspartate aminotransferase; CoNPs, $\mathrm{Co}_{3} \mathrm{O}_{4}$ nanoparticles; CRE, creatinine; TBIL, total bilirubin; TP, total protein. 
CoNPs-BSA at $20 \mathrm{mg} \mathrm{kg}^{-1}$. Neither mouse death nor noticeable abnormal behavior was observed. Serum biochemistry assays and complete blood panel tests were carried out on CoNPs-BSA-injected mice at 1 day, 7 days, 30 days, and 60 days postinjection. The liver function indices, including ALT, AST, TBIL, ALB, TP, and kidney function of CRE were all normal, which suggested no hepatic or kidney function induced by CoNPs-BSA (Figure 5C and D). Histological examination by H\&E staining also showed no appreciable adverse effect of CoNPs-BSA to examine major organs, including the liver, spleen, kidney, lung, and heart at 1 day, 7 days, 30 days, and 60 days postinjection (Figure 5E). Our results suggested that CoNPs-BSA was low toxic to living system at our tested dose and has the potential to serve as promising biomaterials.

\section{Conclusion}

In this work, we optimized a novel artificially controlled degradable CoNPs-BSA for drug delivery and controllable release, as well as MRI-guided photothermal-chemo synergistic cancer therapy. With high photothermal conversion efficiency $(46.19 \%)$, transversal relaxivity $\left(\mathrm{r}_{2}, 5.99 \mathrm{mM}^{-1} \mathrm{~s}^{-1}\right)$, drug encapsulation efficiency (up to 43.20\%), and AAtriggered degradation-induced controllable drug release, the CoNPs-BSA have great potential in cancer theranostics. It is noteworthy that a contrast switch from $T_{2}$ to $T_{1}$ can be observed within the degradation process due to the production of cobalt(II) ions. Therefore, MRI can be used for monitoring the degradation and drug release. This successful in vivo experiment also provided solid evidence on the highly efficient synergistic cancer theranostics. With outstanding diagnosis and therapeutic properties, as well as low toxicity, it can be claimed that the artificially controlled degradable nanomaterials can be a promising candidate for controllable drug release and also provide a novel strategy for designation of the next-generation nanoplatform for synergistic cancer theranostics.

\section{Acknowledgments}

The authors thank the funding of National Natural Science Foundation of China (grant nos. 81573026 and 21301121), Beijing Talent Foundation Outstanding Young Individual Project (2015000026833ZK02), The Joint Foundation Program of Beijing Municipal Natural Science Foundation and Beijing Municipal Education Commission (KZ201810028045), Capacity Building for Sci-Tech Innovation-Fundamental Scientific Research Funds $(025185305000 / 195)$, Project of High-level Teachers in
Beijing Municipal Universities in the Period of 13th Fiveyear Plan (IDHT20180517), Project of Construction of Scientific Research Base by the Beijing Municipal Education Commission, Yanjing Young Scholar Development Program of Capital Normal University, and Youth Innovative Research Team of Capital Normal University.

\section{Author contributions}

Yang Liu and Jing Zhou supervised this manuscript. Tianyang Yun, Yuxin Liu, Yang Liu, and Jing Zhou conceived the project. Tianyang Yun and Yuxin Liu designed the experiments. Yuxin Liu and Qi Jia synthesized and characterized the CoNPs-DOX and in vitro experiments. Tianyang Yun and Yuxin Liu determined MRI in vitro. Tianyang Yun and Shaoqiong Yi performed the in vivo experiments and analyzed the results. All authors wrote the manuscript. All authors contributed toward data analysis, drafting and revising the paper and agree to be accountable for all aspects of the work.

\section{Disclosure}

The authors report no conflicts of interest in this work.

\section{References}

1. Savla R, Minko T. Nanoparticle design considerations for molecular imaging of apoptosis: Diagnostic, prognostic, and therapeutic value. Adv Drug Deliv Rev. 2017;113:122-140.

2. Qian X, Han X, Chen Y. Insights into the unique functionality of inorganic micro/nanoparticles for versatile ultrasound theranostics. Biomaterials. 2017;142:13-30.

3. Fan W, Bu W, Shi J. On The Latest Three-Stage Development of Nanomedicines based on Upconversion Nanoparticles. Adv Mater. 2016; 28(21):3987-4011.

4. Liu Y, Jia Q, Zhou J. Recent Advance in Near-Infrared (NIR) Imaging Probes for Cancer Theranostics. Adv Therap. 2018.

5. Kang T, Li F, Baik S, Shao W, Ling D, Hyeon T. Surface design of magnetic nanoparticles for stimuli-responsive cancer imaging and therapy. Biomaterials. 2017;136:98-114.

6. Liu Y, Li L, Guo Q, et al. Novel Cs-Based Upconversion Nanoparticles as Dual-Modal CT and UCL Imaging Agents for Chemo-Photothermal Synergistic Therapy. Theranostics. 2016;6(10):1491-1505.

7. Huang W, Huang YY, You YY, Nie TQ, Chen TF. High-Yield Synthesis of Multifunctional Tellurium Nanorods to Achieve Simultaneous Chemo-Photothermal Combination Cancer Therapy. Adv Funct Mater. 2017;27(33):1703313.

8. Wang L, Gao C, Liu K, et al. Cypate-Conjugated Porous Upconversion Nanocomposites for Programmed Delivery of Heat Shock Protein 70 Small Interfering RNA for Gene Silencing and Photothermal Ablation. Adv Funct Mater. 2016;26(20):3480-3489.

9. Dai W, Wang X, Song G, et al. Combination antitumor therapy with targeted dual-nanomedicines. Adv Drug Deliv Rev. 2017;115:23-45.

10. Li X, Zhao T, Lu Y. Degradation-Restructuring Induced Anisotropic Epitaxial Growth for Fabrication of Asymmetric Diblock and Triblock Mesoporous Nanocomposites. Adv Mater. 2017;29(30):1701652.

11. Chen Y, Song G, Dong Z, et al. Drug-Loaded Mesoporous Tantalum Oxide Nanoparticles for Enhanced Synergetic Chemoradiotherapy with Reduced Systemic Toxicity. Small. 2017;13(8):1602869. 
12. Liu JJ, Chen Q, Zhu WW. Nanoscale-Coordination-Polymer-Shelled Manganese Dioxide Composite Nanoparticles: A Multistage Redox/ pH/H2O2-Responsive Cancer Theranostic Nanoplatform. Adv Funct Mater. 2017;27(10):1605926.

13. Liu C, Zhang Y, Liu M, et al. A NIR-controlled cage mimicking system for hydrophobic drug mediated cancer therapy. Biomaterials. 2017;139:151-162.

14. Dorozhkin SV. Calcium Orthophosphate-Containing Biocomposites and Hybrid Biomaterials for Biomedical Applications. J Funct Biomater. 2015;6(3):708-832.

15. Guo L, Yan DD, Yang D, et al. Combinatorial photothermal and immuno cancer therapy using chitosan-coated hollow copper sulfide nanoparticles. ACS Nano. 2014;8(6):5670-5681.

16. Song GS, Hao JL, Liang C. Degradable Molybdenum Oxide Nanosheets with Rapid Clearance and Efficient Tumor Homing Capabilities as a Therapeutic Nanoplatform. Angew Chem Int Ed. 2016;55(6): 2122-2126.

17. Chen Y, Cheng L, Dong Z, et al. Degradable Vanadium Disulfide Nanostructures with Unique Optical and Magnetic Functions for Cancer Theranostics. Angew Chem Int Ed Engl. 2017;56(42):12991-12996.

18. Hayashi K, Maruhashi T, Nakamura M, Sakamoto W, Yogo T. One-Pot Synthesis of Dual Stimulus-Responsive Degradable Hollow Hybrid Nanoparticles for Image-Guided Trimodal Therapy. Adv Funct Mater. 2016;26(47):8613-8622.

19. Zhang J, Zhang J, Li W, et al. Degradable Hollow Mesoporous Silicon/ Carbon Nanoparticles for Photoacoustic Imaging-Guided Highly Effective Chemo-Thermal Tumor Therapy in Vitro and in Vivo. Theranostics. 2017;7(12):3007-3020.

20. Liu Y, Zhang G, Guo Q, et al. Artificially controlled degradable inorganic nanomaterial for cancer theranostics. Biomaterials. 2017;112 204-217.

21. Sato P, Nishikimi M, Udenfriend S. Is L-gulonolactone-oxidase the only enzyme missing in animals subject to scurvy? Biochem Biophys Res Commun. 1976;71(1):293-299.

22. Nishikimi M, Kawai T, Yagi K. Guinea pigs possess a highly mutated gene for L-gulono-gamma-lactone oxidase, the key enzyme for L-ascorbic acid biosynthesis missing in this species. J Biol Chem. 1992; 267(30):21967-21972.

23. Bardhan R, Lal S, Joshi A, Halas NJ. Theranostic nanoshells: from probe design to imaging and treatment of cancer. Acc Chem Res. 2011; 44(10):936-946.

24. Liu Y, Guo Q, Zhu X, et al. Optimization of Prussian Blue Coated NaDyF4: $\mathrm{x} \%$ Lu Nanocomposites for Multifunctional Imaging-Guided Photothermal Therapy. Adv Funct Mater. 2016;26(28):5120-5130.

25. Liu Y, Fan H, Guo Q, Jiang A, Du X, Zhou J. Ultra-small pH-responsive Nd-doped $\mathrm{NaDyF}_{4}$ Nanoagents for Enhanced Cancer Theranostic by in situ Aggregation. Theranostics. 2017;7(17):4217-4228.

26. Zhang X, Blasiak B, Marenco AJ, Trudel S, Tomanek B, van VeggelFCJM. Design and Regulation of NaHoF4 and NaDyF4 Nanoparticles for High-Field Magnetic Resonance Imaging. Chem Mater. 2016;28(9): 3060-3072.

27. Datt G, Sen Bishwas M, Manivel Raja M, Abhyankar AC. Observation of magnetic anomalies in one-step solvothermally synthesized nickelcobalt ferrite nanoparticles. Nanoscale. 2016;8(9):5200-5213.
28. Qiao H, Wang Y, Zhang R, et al. MRI/optical dual-modality imaging of vulnerable atherosclerotic plaque with an osteopontin-targeted probe based on $\mathrm{Fe}_{3} \mathrm{O}_{4}$ nanoparticles. Biomaterials. 2017;112:336-345.

29. Mcdonagh BH, Singh G, Hak S, et al. L-DOPA-Coated Manganese Oxide Nanoparticles as Dual MRI Contrast Agents and Drug-Delivery Vehicles. Small. 2016;12(3):301-306.

30. Yang Y, Chao Y, Liu J, et al. Core-shell and co-doped nanoscale metalorganic particles (NMOPs) obtained via post-synthesis cation exchange for multimodal imaging and synergistic thermo-radiotherapy. NPG Asia Mater. 2017;9(1):e344.

31. Sathya A, Guardia P, Brescia R, et al. CoxFe3-xO4 Nanocubes for Theranostic Applications: Effect of Cobalt Content and Particle Size. Chem Mat. 2016;28(6):1769-1780.

32. O'Neill ES, Kaur A, Bishop DP, et al. Hypoxia-Responsive Cobalt Complexes in Tumor Spheroids: Laser Ablation Inductively Coupled Plasma Mass Spectrometry and Magnetic Resonance Imaging Studies. Inorg Chem. 2017;56(16):9860-9868.

33. Lu LT, Dung NT, Tung Led, et al. Synthesis of magnetic cobalt ferrite nanoparticles with controlled morphology, monodispersity and composition: the influence of solvent, surfactant, reductant and synthetic conditions. Nanoscale. 2015;7(46):19596-19610.

34. Liu Y, Jiang A, Jia Q, et al. Rationally designed upconversion nanoprobe for simultaneous highly sensitive ratiometric detection of fluoride ions and fluorosis theranostics. Chem Sci. 2018;9(23):5242-5251.

35. Cheng L, Gong H, Zhu W, et al. PEGylated Prussian blue nanocubes as a theranostic agent for simultaneous cancer imaging and photothermal therapy. Biomaterials. 2014;35(37):9844-9852.

36. Guo Q, Liu Y, Jia Q, et al. Ultrahigh Sensitivity Multifunctional Nanoprobe for the Detection of Hydroxyl Radical and Evaluation of Heavy Metal Induced Oxidative Stress in Live Hepatocyte. Anal Chem. 2017; 89(9):4986-4993.

37. Liu Y, Jia Q, Guo Q, Wei W, Zhou J. Simultaneously activating highly selective ratiometric MRI and synergistic therapy in response to intratumoral oxidability and acidity. Biomaterials. 2018;180:104-116.

38. Feng L, Gai S, He F, et al. Multifunctional mesoporous $\mathrm{ZrO}_{2}$ encapsulated upconversion nanoparticles for mild NIR light activated synergistic cancer therapy. Biomaterials. 2017;147:39-52.

39. Muddineti OS, Kumari P, Ghosh B, Torchilin VP, Biswas S. d- $\alpha-$ Tocopheryl Succinate/Phosphatidyl Ethanolamine Conjugated Amphiphilic Polymer-Based Nanomicellar System for the Efficient Delivery of Curcumin and To Overcome Multiple Drug Resistance in Cancer. ACS Appl Mater Interfaces. 2017;9(20):16778-16792.

40. Li W, Zhang H, Guo X, et al. Gold Nanospheres-Stabilized Indocyanine Green as a Synchronous Photodynamic-Photothermal Therapy Platform That Inhibits Tumor Growth and Metastasis. ACS Appl Mater Interfaces. 2017;9(4):3354-3367.

41. Fang J, Tsukigawa K, Liao L, Yin H, Eguchi K, Maeda H. Styrenemaleic acid-copolymer conjugated zinc protoporphyrin as a candidate drug for tumor-targeted therapy and imaging. J Drug Target. 2016; 24(5):399-407.
International Journal of Nanomedicine

\section{Publish your work in this journal}

The International Journal of Nanomedicine is an international, peerreviewed journal focusing on the application of nanotechnology in diagnostics, therapeutics, and drug delivery systems throughout the biomedical field. This journal is indexed on PubMed Central, MedLine, CAS, SciSearch ${ }^{\circledR}$, Current Contents ${ } /$ Clinical Medicine,

\section{Dovepress}

Journal Citation Reports/Science Edition, EMBase, Scopus and the Elsevier Bibliographic databases. The manuscript management system is completely online and includes a very quick and fair peer-review system, which is all easy to use. Visit http://www.dovepress.com/ testimonials.php to read real quotes from published authors. 